\title{
The one-sided ergodic Hilbert transform in Banach spaces
}

\author{
by \\ Guy Cohen (Beer Sheva), Christophe Cuny (Nouméa) \\ and Michael Lin (Beer Sheva)
}

\begin{abstract}
Let $T$ be a power-bounded operator on a (real or complex) Banach space. We study the convergence of the one-sided ergodic Hilbert transform $\lim _{n} \sum_{k=1}^{n} \frac{T^{k} x}{k}$. We prove that weak and strong convergence are equivalent, and in a reflexive space also $\sup _{n}\left\|\sum_{k=1}^{n} \frac{T^{k} x}{k}\right\|<\infty$ is equivalent to the convergence. We also show that $-\sum_{k=1}^{\infty} \frac{T^{k}}{k}$ (which converges on $(I-T) X$ ) is precisely the infinitesimal generator of the semigroup $(I-T)^{r} \mid \overline{(I-T) X}$.
\end{abstract}

1. Introduction. Izumi 12 raised the question of the a.e. convergence of the one-sided ergodic Hilbert transform (EHT) $\sum_{k=1}^{\infty} \frac{f \circ \theta^{k}}{k}$ associated to a probability preserving transformation $\theta$ and functions in $L_{2}(S, \Sigma, \mu)$ (which, by Kronecker's lemma, would be a strengthening of Birkhoff's pointwise ergodic theorem). Halmos [10] proved that for every ergodic probability preserving transformation on a non-atomic space there always exists a centred $f \in L_{2}$ such that the one-sided EHT fails to converge in $L_{2}$-norm.

On the other hand, Cotlar [5] proved that when $T$ is the operator induced by an invertible probability preserving transformation, for every $f \in L_{1}$ the two-sided EHT $\sum_{k=1}^{\infty} \frac{T^{k} f-T^{-k} f}{k}$ converges a.e., and in $L_{p}$-norm when $f \in L_{p}$, $1 \leq p<\infty$. Campbell 2 proved that for a unitary operator $T$ on a complex Hilbert space $H$ the two-sided EHT converges in norm for every $f \in H$.

For $T$ unitary on a complex Hilbert space $H$, Gaposhkin [9] obtained a spectral characterization of the norm convergence of the one-sided EHT $\sum_{k=1}^{\infty} \frac{T^{k} f}{k}$. For a normal contraction $T$, several additional characterizations were recently obtained by Cohen and Lin [3, who proved that norm convergence is equivalent to weak convergence; this had been proved by Assani and Lin [1] for $T$ unitary or self-adjoint.

2010 Mathematics Subject Classification: Primary 47A35, 28D05, 37A05; Secondary $47 \mathrm{~B} 38$.

Key words and phrases: ergodic Hilbert transform, operator power series, measure preserving transformations, semigroup of fractional powers. 
Recently, Cuny [6] proved that for $T$ a Dunford-Schwartz operator on a $\sigma$-finite measure space or a positive contraction of an $L_{p}$ space, $1<$ $p<\infty$, norm convergence of $\sum_{k=1}^{\infty} \frac{T^{k} f}{k}$ implies a.e. convergence. For additional results and references concerning pointwise convergence see [9] and [1].

In this paper we consider a power-bounded operator $T$ on a Banach space $X$ (which is therefore a contraction in an equivalent norm), and for $x \in X$ we study the convergence of the series

$$
\sum_{k=1}^{\infty} \frac{T^{k} x}{k}
$$

Obviously, weak convergence of (1) implies that $x^{*}(x)=0$ whenever $T^{*} x^{*}=$ $x^{*}$, so necessarily $x \in \overline{(I-T) X}$ (e.g. [13, p. 73]). Derriennic and Lin [7, Theorem 2.23] proved that the series (1) converges in norm for every $x$ in $\overline{(I-T) X}$ if and only if $(I-T) X$ is closed. It was noted in [3] that the same holds if norm convergence is replaced by weak convergence.

We obtain several necessary and sufficient conditions for the convergence of the one-sided ergodic Hilbert transform (1). One of our main results is that weak convergence of the series (1) always implies its norm convergence.

2. On some operator series. Motivated by conditions for the central limit theorem for stationary ergodic Markov chains, Derriennic and Lin [7] defined for $0<\alpha<1$ and $T$ power-bounded on $X$ the operator $(I-T)^{\alpha}$ by the series $(I-T)^{\alpha}=I-\sum_{n=1}^{\infty} a_{n}^{(\alpha)} T^{n}$, where the coefficients are those of the expansion of $(1-t)^{\alpha}$ in the interval $[-1,1]$, with $a_{n}^{(\alpha)}>0$ and $\sum_{n=1}^{\infty} a_{n}^{(\alpha)}=1$. It is not difficult to show that $(I-T)^{\alpha} X \subset \overline{(I-T) X}$. It is proved in [7] that when $T$ is mean-ergodic, $x \in(I-T)^{\alpha} X$ if and only if the series $\sum_{n=1}^{\infty} \frac{T^{n} x}{n^{1-\alpha}}$ converges strongly. The proof uses the series representation of $1 /(1-t)^{\alpha}$, which converges for $|t|<1$. This suggests the idea in [6] that in order to study the one-sided EHT, we try to connect it with the inverse of an analytic function on the open unit disk $D:=\{z \in \mathbb{C}:|z|<1\}$. For $z \in D$, define

$$
H(z):=\log \left(\frac{\mathrm{e}}{1-z}\right)=1+\sum_{n \geq 1} \frac{z^{n}}{n}=\sum_{n \geq 0} \beta_{n} z^{n} .
$$

Since $1-\mathrm{e} \notin D, H \neq 0$ on $D$. Hence $G:=1 / H$ is well defined and analytic on $D$, so there exists $\left\{\alpha_{n}\right\}_{n \geq 0}$ such that

$$
G(z)=\sum_{n \geq 0} \alpha_{n} z^{n} \quad \forall z \in D .
$$

One can see that $\alpha_{0}=1$, and it follows from the identity $G(z) H(z)=1$ that

$$
\beta_{n}+\sum_{k=1}^{n} \alpha_{k} \beta_{n-k}=\beta_{n}+\sum_{k=0}^{n-1} \beta_{k} \alpha_{n-k}=0 \quad \forall n \geq 1 .
$$


Consequently, the coefficients $\left\{\alpha_{n}\right\}$ are real, and it follows from 17, Theorem 2.31, p. 192] that $\alpha_{n} \sim-1 /\left(n(\log n)^{2}\right)$. For convenience we denote $\gamma_{n}:=K /\left(n(\log (n+1))^{2}\right), n \geq 1$, where $K:=\sup _{n \geq 1} n(\log (n+1))^{2}\left|\alpha_{n}\right|$, so that $\left|\alpha_{n}\right| \leq \gamma_{n}$ for $n \geq 1$. Since $G(t)=1 / H(t)$ for $t \in(0,1)$ and $G(1)$ is defined by an absolutely convergent power series, letting $t \rightarrow 1^{-}$we obtain $G(1)=0$.

We now fix a power-bounded operator $T$ on a (real or complex) Banach space $X$. Since $\sum_{n>0}\left|\alpha_{n}\right|<\infty$ and the coefficients are real, the operator series $\sum_{n \geq 0} \alpha_{n} T^{n}$ converges in operator norm, and defines a bounded operator on $X$, denoted by $G(T)$.

For every $n \geq 1$ we define

$$
H_{n}(T):=I+\sum_{k=1}^{n} \frac{T^{k}}{k}=\sum_{k=0}^{n} \beta_{k} T^{k}
$$

and put $H(T) x=\lim _{n \rightarrow \infty} H_{n}(T) x$ whenever the limit exists in norm.

Proposition 2.1. Let $T$ be a power-bounded operator on a Banach space $X$. Then

$$
\sup _{n \geq 1}\left\|H_{n}(T) G(T)\right\|<\infty .
$$

Proof. Since the series $\sum_{n \geq 0} \alpha_{n}$ is absolutely convergent, we have

$$
\begin{aligned}
H_{n} & (T) G(T)=\sum_{k=0}^{n} \beta_{k} T^{k}+\sum_{k=0}^{n} \beta_{k} \sum_{m \geq 1} \alpha_{m} T^{m+k} \\
& =\sum_{k=0}^{n} \beta_{k} T^{k}+\sum_{k=0}^{n} \beta_{k} \sum_{m \geq k+1} \alpha_{m-k} T^{m} \\
& =\sum_{k=0}^{n} \beta_{k} T^{k}+\sum_{m=1}^{n}\left(\sum_{k=0}^{m-1} \beta_{k} \alpha_{m-k}\right) T^{m}+\sum_{m \geq n+1}\left(\sum_{k=0}^{n} \beta_{k} \alpha_{m-k}\right) T^{m} \\
& =I+\sum_{m \geq n+1}\left(\sum_{k=0}^{n} \beta_{k} \alpha_{m-k}\right) T^{m},
\end{aligned}
$$

where we used $\beta_{0}=1$ and (2) for the last equality. It suffices to deal with the series of the last equality. We have

$$
\begin{aligned}
\sum_{m \geq n+1} & \left(\sum_{k=0}^{n} \beta_{k} \alpha_{m-k}\right) T^{m} \\
& =\sum_{m=n+1}^{2 n}\left(\sum_{k=0}^{n} \beta_{k} \alpha_{m-k}\right) T^{m}+\sum_{m \geq 2 n+1}\left(\sum_{k=0}^{n} \beta_{k} \alpha_{m-k}\right) T^{m} .
\end{aligned}
$$


For the last sum, monotonicity of $\left\{\gamma_{n}\right\}$ and $\sup _{n}\left\|T^{n}\right\|=M<\infty$ yield

$$
\begin{aligned}
\| \sum_{m \geq 2 n+1} & \left(\sum_{k=0}^{n} \beta_{k} \alpha_{m-k}\right) T^{m}\left\|\leq \sum_{m \geq 2 n+1} \gamma_{m-n}\left(\sum_{k=0}^{n} \beta_{k}\right)\right\| T^{m} \| \\
& \leq M(2+\log n) \sum_{m \geq 2 n+1} \gamma_{m-n} \leq C \log n \sum_{j \geq n+1} \frac{K}{j(\log (j+1))^{2}} \leq C^{\prime} .
\end{aligned}
$$

Let us deal with the first sum on the right-hand side of (6). By (2), we have

$$
\begin{aligned}
\left\|\sum_{m=n+1}^{2 n}\left(\sum_{k=0}^{n} \beta_{k} \alpha_{m-k}\right) T^{m}\right\| & =\left\|\sum_{m=n+1}^{2 n} \beta_{m} T^{m}+\sum_{m=n+1}^{2 n}\left(\sum_{k=n+1}^{m-1} \beta_{k} \alpha_{m-k}\right) T^{m}\right\| \\
& \leq \sum_{m=n+1}^{2 n} \beta_{m}\left\|T^{m}\right\|+\sum_{m=n+1}^{2 n}\left(\sum_{k=n+1}^{m-1} \beta_{k} \gamma_{m-k}\right)\left\|T^{m}\right\| \\
& \leq M n \frac{1}{n+1}+M n \frac{1}{n+1} \sum_{j \geq 1} \gamma_{j},
\end{aligned}
$$

using the definition of $\left\{\beta_{n}\right\}$ and its monotonicity for the last inequality.

The next proposition provides the main tool for our results. It was proved in [6] in the context of power-bounded operators on $L_{p}$ spaces.

Proposition 2.2. Let $T$ be a power-bounded operator on a Banach space $X$ and let $x \in \overline{(I-T) X}$. Then

$$
\lim _{n \rightarrow \infty}\left\|x-H_{n}(T) G(T) x\right\|=0 .
$$

Proof. By the previous proposition, it is enough to prove the convergence for $x \in(I-T) X$. By (5), the assertion is that for $x \in(I-T) X$,

$$
\left\|\sum_{m \geq n+1}\left(\sum_{k=0}^{n} \beta_{k} \alpha_{m-k}\right) T^{m} x\right\| \stackrel{n \rightarrow \infty}{\longrightarrow} 0 .
$$

We denote $M:=\sup _{n \geq 0}\left\|T^{n}\right\|$. For $u \in X$ we have

$$
\begin{aligned}
\sum_{m \geq n+1} & \left(\sum_{k=0}^{n} \beta_{k} \alpha_{m-k}\right) T^{m}(u-T u) \\
= & \sum_{m \geq n+1}\left(\sum_{k=0}^{n} \beta_{k} \alpha_{m-k}\right) T^{m} u-\sum_{m \geq n+2}\left(\sum_{k=0}^{n} \beta_{k} \alpha_{m-k-1}\right) T^{m} u \\
= & \sum_{m \geq n+2}\left(\sum_{k=1}^{n}\left(\beta_{k}-\beta_{k-1}\right) \alpha_{m-k}\right) T^{m} u+\left(\sum_{k=0}^{n} \beta_{k} \alpha_{n+1-k}\right) T^{n+1} u \\
& +\sum_{m \geq n+1} \alpha_{m} T^{m} u-\sum_{m \geq n+2} \beta_{n} \alpha_{m-n-1} T^{m} u .
\end{aligned}
$$


We estimate the norms of the last three terms using power-boundedness, (2) and monotonicity of $\left\{\beta_{n}\right\}$ and obtain

$$
\begin{aligned}
& \left\|\sum_{k=0}^{n} \beta_{k} \alpha_{n+1-k} T^{n+1} u+\sum_{m \geq n+1} \alpha_{m} T^{m} u-\sum_{m \geq n+2} \beta_{n} \alpha_{m-n-1} T^{m} u\right\| \\
& \leq M\|u\|\left(\sum_{j=[n / 2]+1}^{n+1} \gamma_{j}+\frac{1}{[n / 2]+1} \sum_{j=1}^{[n / 2]+1} \gamma_{j}+\sum_{m \geq n+1} \gamma_{m}+\frac{1}{n} \sum_{m \geq 1} \gamma_{m}\right) \stackrel{n \rightarrow \infty}{\longrightarrow} 0 .
\end{aligned}
$$

It remains to deal with the first term on the right-hand side of (7). Splitting the inner sum according to $k \leq[n / 2]$ and using monotonicity of $\left\{\gamma_{n}\right\}$, we obtain

$$
\begin{array}{r}
\left\|\sum_{m \geq n+2}\left(\sum_{k=1}^{n}\left(\beta_{k}-\beta_{k-1}\right) \alpha_{m-k}\right) T^{m} u\right\| \leq M \sum_{m \geq n+2}\left(\sum_{k=1}^{n}\left|\beta_{k}-\beta_{k-1}\right| \gamma_{m-k}\right)\|u\| \\
\leq M \sum_{k=1}^{[n / 2]}\left(\beta_{k-1}-\beta_{k}\right)\|u\| \sum_{m \geq n+2} \gamma_{m-[n / 2]}+\frac{M\|u\|}{[n / 2]} \sum_{m \geq n+2} \gamma_{m-n} \\
\leq \frac{K M}{\log n}\|u\|,
\end{array}
$$

for a constant $K>0$ independent of $u$ (and $T$ ), which proves the proposition.

3. Convergence of the one-sided ergodic Hilbert transform. In this section we obtain several criteria for the convergence of the one-sided ergodic Hilbert transform of a power-bounded operator. Additional criteria are obtained when the Banach space is reflexive. For normal contractions in a complex Hilbert space the main theorems were proved in [3]; for unitary operators and self-adjoint contractions they had been obtained in [1.

Lemma 3.1. Let $T$ be a power-bounded operator on a Banach space $X$ and let $x \in X$. If $\liminf _{n \rightarrow \infty}\left\|\sum_{k=1}^{n} \frac{T^{k} x}{k}\right\|<\infty$, then $x \in \overline{(I-T) X}$.

Proof. The condition implies that $\sup _{j}\left\|H_{n_{j}}(T) x\right\|<\infty$ for some $\left\{n_{j}\right\}$. Hence $x^{*}(x)=0$ whenever $T^{*} x^{*}=x^{*}$, which implies $x \in \overline{(I-T) X}$.

THEOREM 3.2. Let $T$ be a power-bounded operator on a Banach space $X$ and let $x \in X$. Then the following are equivalent:

(i) There is an increasing $\left\{n_{j}\right\}$ such that $\sum_{k=1}^{n_{j}} \frac{T^{k} x}{k}$ converges weakly.

(ii) The series $\sum_{k=1}^{\infty} \frac{T^{k} x}{k}$ converges weakly.

(iii) The series $\sum_{k=1}^{\infty} \frac{T^{k} x}{k}$ converges in norm.

Proof. Obviously we have to prove only that (i) implies (iii). We assume that $H_{n_{j}}(T) x$ converges weakly to some $z$; this implies that $\left\{\left\|H_{n_{j}}(T) x\right\|\right\}$ 
is bounded, so $x$ (by Lemma 3.1), and hence $z$, are in $\overline{(I-T) X}$ (since it is weakly closed [8, Theorem V.3.13]), and Proposition [2.2 yields $x=$ $H(T) G(T) x$. Using weak continuity of $G(T)$ we then obtain

$$
\begin{aligned}
G(T) z & =\text { weak- } \lim G(T) H_{n_{j}}(T) x \\
& =\text { weak- } \lim H_{n_{j}}(T) G(T) x=H(T) G(T) x=x .
\end{aligned}
$$

Since $z \in \overline{(I-T) X}$, Proposition 2.2 implies that $H_{n}(T) x=H_{n}(T) G(T) z$ converges in norm (to $z$ ).

REMARK. The theorem is the analogue, for the one-sided EHT, of Yosida's theorem for the Cesàro averages (e.g. [13, p. 72]).

Proposition 3.3. Let $T$ be power-bounded on $X$. The series $\sum_{k=1}^{\infty} \frac{T^{k} x}{k}$ converges if and only if $x \in G(T)[\overline{(I-T) X}]$.

Proof. Denote $X_{0}:=G(T)[\overline{(I-T) X}]$. Proposition 2.2 shows that the limit operator $H(T)$, defined for all those $x$ for which the one-sided EHT converges (in norm), has $X_{0}$ included in its domain of definition, and $H(T)$ maps its domain of definition onto all of $\overline{(I-T) X}$. By the previous proof, convergence of $H_{n}(T) x$ implies that $x \in X_{0}$, so $X_{0}$ is the precise domain of definition of the one-sided EHT.

REMARK. Note that when $T$ is mean ergodic, the ergodic decomposition yields $X_{0}=G(T) X$, since $G(1)=0$ implies $G(T) z=0$ whenever $T z=z$.

Corollary 3.4. Let $T$ be power-bounded on $X$. For $0<\delta<1$ we have $(I-T) X \subset(I-T)^{\delta} X \subset G(T)[\overline{(I-T) X}] \subset G(T) X \subset \overline{(I-T) X}$

Proof. Combining (4) with Lemma 3.1 we obtain $G(T) X \subset \overline{(I-T) X}$. The first inclusion and the convergence of $H_{n}(T) x$ for $x \in(I-T)^{\delta} X$ are proved in [7].

Remark. When $(I-T) X$ is not closed, $(I-T)^{\delta_{1}} X \subsetneq(I-T)^{\delta_{2}} X$ for every $\delta_{2}<\delta_{1} \leq 1$ by [7], hence $(I-T)^{\delta} X \neq G(T)[\overline{(I-T) X}]$ for $0<\delta \leq 1$. By [7, Theorem 2.23], $G(T)[\overline{(I-T) X}] \neq \overline{(I-T) X}$ in this case.

Proposition 3.5. Let $X=Y^{*}$ be a dual Banach space and let $T=S^{*}$ be a power-bounded dual operator on $X$. If

$$
\liminf _{n \rightarrow \infty}\left\|\sum_{k=1}^{n} \frac{T^{k} x}{k}\right\|<\infty
$$

then $x \in G(T) X$. If in addition $T$ is mean ergodic, then $\sum_{k=1}^{\infty} \frac{T^{k} x}{k}$ converges in norm.

Proof. By Lemma 3.1. (8) implies that $x \in \overline{(I-T) X}$. By assumption we have an increasing $\left\{n_{j}\right\}$ such that $\sup _{j \geq 1}\left\|\sum_{k=1}^{n_{j}} \frac{T^{k} x}{k}\right\|<\infty$. Let LIM 
denote a fixed Banach limit (e.g. [13, p. 135]), and for $f \in Y$ ( $Y$ is the predual of $X$ ) define

$\psi(f)=\operatorname{LIM}\left\{\sum_{k=1}^{n_{j}} \frac{x\left(S^{k} f\right)}{k}\right\}=\operatorname{LIM}\left\{\sum_{k=1}^{n_{j}} \frac{\left(T^{k} x\right)(f)}{k}\right\}=\operatorname{LIM}\left\{\left[H_{n_{j}}(T) x\right](f)\right\}$.

By (8), $\psi$ is well-defined, linear and bounded, so $\psi \in Y^{*}=X$. Since $x$ is in $\overline{(I-T) X}$, we have $G(T) H_{n}(T) x=H_{n}(T) G(T) x \rightarrow x$ in norm, by Proposition 2.2. Since Banach limits preserve convergence and $G(T)=G(S)^{*}$, we obtain

$$
\begin{aligned}
{[G(T) \psi](f) } & =\psi(G(S) f)=\operatorname{LIM}\left\{\left[H_{n_{j}}(T) x\right](G(S) f)\right\} \\
& =\operatorname{LIM}\left\{\left[G(T) H_{n_{j}}(T) x\right](f)\right\}=x(f)
\end{aligned}
$$

for every $f \in Y$, so $G(T) \psi=x$.

If in addition $T$ is mean ergodic, then $x \in G(T) X=G(T) \overline{(I-T) X}=X_{0}$, so $H_{n}(T) x$ converges by Proposition 3.3 .

REMARK. The use of a Banach limit is not necessary when the predual $Y$ is separable; in this case the weak-* topology on bounded sets in $X=Y^{*}$ is metrizable, and there exists an increasing sequence of integers $n_{j}$ such that $H_{n_{j}}(T) x$ converges weak-* to some $\psi \in X$. Since $x \in \overline{(I-T) X}$, weak-* continuity of $G(T)$ and Proposition 2.2 imply that $G(T) \psi=$ weak-* $\lim G(T) H_{n_{j}}(T) x=x$.

COROLlary 3.6. Let $T$ be a power-bounded operator on a reflexive $B a$ nach space. Then $\sum_{k=1}^{\infty} \frac{T^{k} x}{k}$ converges in norm if and only if

$$
\liminf _{n \rightarrow \infty}\left\|\sum_{k=1}^{n} \frac{T^{k} x}{k}\right\|<\infty
$$

Proof. Since $X$ is reflexive, $T$ is a dual mean ergodic operator.

Combining the previous corollary with [6, Theorem 1.3] we obtain the following.

Corollary 3.7. Let $1<p<\infty$ and let $T$ be a positive contraction of $L_{p}(S, \Sigma, \mu)$ of a $\sigma$-finite measure space. If $f \in L_{p}$ satisfies

$$
\liminf _{n \rightarrow \infty}\left\|\sum_{k=1}^{n} \frac{T^{k} f}{k}\right\|_{p}<\infty
$$

then $\sum_{k=1}^{n} \frac{T^{k} f}{k}$ converges almost everywhere and

$$
\sup _{n \geq 1}\left|\sum_{k=1}^{n} \frac{T^{k} f}{k}\right| \in L_{p}(S, \mu) .
$$

Remark. Positivity is not needed when $T$ is a Dunford-Schwartz operator-we apply [6, Theorem 1.2] instead of [6, Theorem 1.3]. 
Example 1 (A dual operator $T$ and $x \in X$ with $\left\{H_{n}(T) x\right\}$ bounded and non-convergent). Define $S$ on $Y=\ell_{1}$ by

$$
S\left(a_{0}, a_{1}, a_{2}, \ldots\right)=\left(a_{1}, a_{2}, a_{3}, \ldots\right) .
$$

Then $T=S^{*}$ on $X=\ell_{\infty}$ is given by

$$
T\left(b_{0}, b_{1}, b_{2}, \ldots\right)=\left(0, b_{0}, b_{1}, b_{2}, \ldots\right) .
$$

For $e=(1,1, \ldots) \in \ell_{\infty}$ we have $\left\|n^{-1} \sum_{k=1}^{n} T^{k} e\right\|_{\infty}=1$ for every $n$, so $T$ is not mean ergodic since it has no fixed points. We put $x=G(T) e$; Proposition 2.1 yields $\sup _{n}\left\|H_{n}(T) x\right\|<\infty$.

We show that $\left\{H_{n}(T) x\right\}$ is not Cauchy. We use $G(1)=0$ and the asymptotic behaviour of $\alpha_{n}$ to conclude that for $m \geq 0$ the $m$ th coordinate of $x=G(T) e$ is

$$
x_{m}=\sum_{k=0}^{m} \alpha_{k}=-\sum_{k \geq m+1} \alpha_{k} \sim \frac{C}{\log (m+2)} .
$$

For $k \geq 0$ the $m$ th coordinate of $T^{k} x$ is 0 for $m<k$ and $x_{m-k}$ for $m \geq k$.

Let $1<p<q$. For $m>q$ we have

$$
\left(\sum_{k=p}^{q} \beta_{k} T^{k} x\right)_{m}=\sum_{k=p}^{q} \beta_{k} x_{m-k} \geq \sum_{k=p}^{q} \frac{C \beta_{k}}{\log (m+2-k)} .
$$

If we take $q=p^{2}$, for $m=p^{2}+p$ we obtain

$$
\begin{aligned}
\left\|H_{q}(T) x-H_{p}(T) x\right\| & \geq \sum_{k=p}^{p^{2}} \frac{C \beta_{k}}{\log (m+2-k)} \geq \frac{C}{\log (p+2)} \sum_{k=p}^{p^{2}} \frac{1}{k} \\
& \geq \frac{K}{\log (p+2)}\left(\log \left(p^{2}\right)-\log p\right) \nrightarrow 0 .
\end{aligned}
$$

Proposition 3.8. Let $T$ be a power-bounded operator on a Banach space $X$ such that $T^{* *}$ is mean ergodic on $X^{* *}$ (e.g. $X$ is reflexive). Then $\sum_{k=1}^{\infty} \frac{T^{k} x}{k}$ converges if and only if $\liminf _{n \rightarrow \infty}\left\|\sum_{k=1}^{n} \frac{T^{k} x}{k}\right\|<\infty$.

Proof. Assume the condition (8) holds. We identify $X$ as a closed subspace of $X^{* *}$ by the canonical embedding, which is $T^{* *}$-invariant.

We apply Proposition 3.5 to $T^{* *}$ and $x$ and obtain the existence of $x^{* *} \in X^{* *}$ such that $G\left(T^{* *}\right) x^{* *}=x$. As noted earlier, the mean ergodicity of $T^{* *}$ implies the existence of $z^{* *} \in \overline{\left(I-T^{* *}\right) X^{* *}}$ such that $G\left(T^{* *}\right) z^{* *}=x$. By Proposition 2.2 we then have

$$
\left\|H_{n}(T) x-z^{* *}\right\|=\left\|H_{n}\left(T^{* *}\right) x-z^{* *}\right\|=\left\|H_{n}\left(T^{* *}\right) G\left(T^{* *}\right) z^{* *}-z^{* *}\right\| \rightarrow 0,
$$

which shows that $z^{* *} \in X$ and proves the desired convergence.

Remark. The proof shows that (8) always implies that $x \in G\left(T^{* *}\right) X^{* *}$,

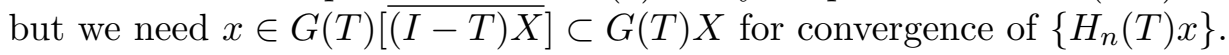


EXAmple 2 (A mean ergodic power-bounded $T$ and $x \in X$ with $\left\{H_{n}(T) x\right\}$ bounded and non-convergent). On $X=c_{0}$ we define the isometry $T\left(b_{0}, b_{1}, b_{2}, \ldots\right)=\left(0, b_{0}, b_{1}, b_{2}, \ldots\right)$,

Since $T^{n} z \rightarrow 0$ weakly for every $z \in c_{0}$, the operator $T$ is mean ergodic. Note that the dual $T^{*}$ on $\ell_{1}$ is the operator $S$ of Example 1, and is also mean ergodic. The operator $T^{* *}$ on $\ell_{\infty}$ is in fact the operator of Example 1, and we put $x=G\left(T^{* *}\right) e$. As shown in Example 1, the $m$ th coordinate of $x$ behaves asymptotically like $C / \log (m+2)$, so $x \in c_{0}$. The computations in Example 1 prove boundedness and non-convergence of $\left\{H_{n}(T) x\right\}$; in particular, $x \notin G(T) X$.

Derriennic and Lin [7] proved that for $T$ power-bounded on $X$ the family of operators $\left\{(I-T)^{r}:=(I-T)^{[r]}(I-T)^{r-[r]}: r \geq 0\right\}$ has the semigroup property, and in [7, Theorem 2.22] it is proved that the restriction of this semigroup to the closed subspace $\overline{(I-T) X}$ is strongly continuous at 0 (with the identity as limit at 0 ). The following answers a question raised in [1].

Theorem 3.9. Let $T$ be power-bounded on $X$, and let $A$ be the infinitesimal generator of the semigroup $\left\{(I-T)^{r}: r \geq 0\right\}$ defined on $\overline{(I-T) X}$. Then $x \in \mathcal{D}(A)$ if and only if the series $\sum_{n=1}^{\infty} \frac{T^{n} x}{n}$ converges. The generator is then given by

$$
A x=-\sum_{n=1}^{\infty} \frac{T^{n} x}{n}, \quad x \in \mathcal{D}(A) .
$$

Proof. If the one-sided EHT converges in norm, then $x \in \mathcal{D}(A)$ and $A x=-\sum_{k=1}^{\infty} \frac{T^{k} x}{k}$, by [7, Proposition 2.21].

Now let $x \in \mathcal{D}(A) \subset \overline{(I-T) X}$. Then $A x \in \overline{(I-T) X}$ and Proposition 2.2 implies that $z:=G(T)(I-A) x$ is in the domain of the one-sided EHT and satisfies $\lim _{n} H_{n}(T) z=(I-A) x$. But since $z$ is in the domain of the EHT, by [7], $z \in \mathcal{D}(A)$ and $\lim \left(H_{n}(T)-I\right) z=-A z$, so $(I-A)(x-z)=0$.

When $A y=y$, the semigroup continuity yields $(I-T)^{r} y=\mathrm{e}^{r} y$ (see the first part of the proof of [15, Theorem IX.4.1, p. 240], which applies also to real Banach spaces), so in particular $(I-T) y=\mathrm{e} y$, implying that if $y \neq 0$ then $\left\|T^{n} y\right\|=(\mathrm{e}-1)^{n}\|y\| \rightarrow \infty$, contradicting the power-boundedness of $T$. Hence $x=z$, so $\sum_{n=1}^{\infty} \frac{T^{n} x}{n}$ converges (to $-A x$ ).

REMARK. If in the proof we use [1, Proposition 4.1] instead of [7], we can obtain Theorem 3.2 as a corollary; we have preferred to prove Theorem 3.2 directly, independently of results from semigroup theory (used in [1] and in Theorem 3.9p.

Given $T$ power-bounded on a Banach space $X$, for every $\epsilon>0$ the series $\sum_{k=1}^{\infty} \frac{T^{k}}{k^{1+\epsilon}}$ converges in operator norm. Combining the theorem with Corollary 4.5 of Assani and Lin [1] we obtain the following. 
COROLlary 3.10. Let $T$ be a power-bounded operator on a Banach space $X$ and let $x \in \overline{(I-T) X}$. Then

$$
\sum_{n=1}^{\infty} \frac{T^{n} x}{n} \text { converges } \Leftrightarrow \lim _{\epsilon \rightarrow 0+} \sum_{k=1}^{\infty} \frac{T^{k} x}{k^{1+\epsilon}} \text { exists }
$$

and the two limits are equal.

The following was proved in [3] for normal contractions in a Hilbert space.

Corollary 3.11. Let $T$ be power-bounded on a reflexive Banach space $X$ and let $x \in \overline{(I-T) X}$. Then

$$
\sum_{n=1}^{\infty} \frac{T^{n} x}{n} \text { converges } \Leftrightarrow \sup _{0<\epsilon<1 / 2}\left\|\sum_{k=1}^{\infty} \frac{T^{k} x}{k^{1+\epsilon}}\right\|<\infty
$$

Proof. We first assume that $\sup _{0<\epsilon<1 / 2}\left\|\sum_{k=1}^{\infty} \frac{T^{k} x}{k^{1+\epsilon}}\right\|<\infty$. By weak sequential compactness of bounded sets, there is a sequence $\epsilon_{j} \rightarrow 0$ such that $\sum_{k=1}^{\infty} \frac{T^{k} x}{k^{1+\epsilon_{j}}}$ converges weakly, say to $z$. By the proof of [1, Corollary 4.5] $\sum_{k=2}^{\infty} \frac{T^{k} x}{k(k-1)^{\epsilon_{j}}}$ converges weakly to $z-T x$. Combining this with Lemma 4.4 of [1] we obtain

$$
\text { weak- } \lim _{\epsilon_{j} \rightarrow 0} \frac{(I-T)^{\epsilon_{j}} x-x}{\epsilon_{j}}=-T x-\text { weak- } \lim _{\epsilon_{j} \rightarrow 0} \sum_{k=2}^{\infty} \frac{T^{k} x}{k(k-1)^{\epsilon_{j}}}=-z .
$$

By a result of Yosida [11, Theorem 10.5.4, p. 318] this already implies $x \in$ $\mathcal{D}(A)$ (and then $A x=-z$ ), so by Theorem 3.9, $\sum_{n=1}^{\infty} \frac{T^{n} x}{n}$ converges strongly.

The converse implication follows from the previous corollary.

4. The one-sided ergodic Hilbert transform of $L_{1}$ isometries. Lin and Sine [14] proved that for $T$ a contraction of $L_{1}(S, \Sigma, \mu)$, a function $f$ is in $(I-T) L_{1}$ if (and obviously only if) $\sup _{n}\left\|\sum_{k=1}^{n} T^{k} f\right\|_{1}<\infty$. Unfortunately, their method cannot be used to prove that for $L_{1}$-contractions, $f \in G(T) L_{1}$ if $\sup _{n}\left\|\sum_{k=1}^{n} \frac{T^{k} f}{k}\right\|_{1}<\infty$, since the coefficients $\left\{\alpha_{n}\right\}_{n \geq 1}$ of $G(z)$ are not all negative (though they eventually are). However, we can still deal with isometries of $L_{1}$.

Recall that for a complete finite measure space, $L_{\infty}^{*}=L_{1}^{* *}$ is identified with the space $b a(S, \Sigma, \mu)$ of bounded finitely additive (signed) measures, called charges (see [8, IV.8.16]), and by the canonical embedding $L_{1}$ is identified with the space $M(S, \Sigma, \mu)$ of countably additive signed measures absolutely continuous with respect to $\mu$. A charge $\eta \in b a(S, \Sigma, \mu)$ is called a pure charge if $|\eta|$ does not bound any non-negative measure, and then $\|\eta+\nu\|=\|\eta\|+\|\nu\|$ for any countably additive $\nu$. Every $\eta \in L_{1}^{* *}$ can be decomposed as $\eta=\eta_{1}+\eta_{0}$ with $\eta_{1}$ countably additive and $\eta_{0}$ a pure 
charge [16. The following lemma must be known (its first part is essentially [16, Theorem 2.6]).

LEMMA 4.1.

(i) Let $\eta \in b a(S, \Sigma, \mu)$ satisfy $\|\eta+\nu\|=\|\eta\|+\|\nu\|$ for every countably additive $\nu$. Then $\eta$ is a pure charge.

(ii) The set of pure charges is norm-closed.

Proof. (i) Write $\eta=\eta_{1}+\eta_{0}$ with $\eta_{1}$ countably additive and $\eta_{0}$ a pure charge. Putting $\nu=-\eta_{1}$ and using the assumption, we obtain

$$
\left\|\eta_{0}\right\|=\|\eta+\nu\|=\|\eta\|+\left\|\eta_{1}\right\|=\left\|\eta_{0}\right\|+2\left\|\eta_{1}\right\|,
$$

which implies $\eta_{1}=0$.

(ii) Let $\left\{\eta_{n}\right\}$ be a sequence of pure charges converging in norm to $\eta$ and use (i).

Lemma 4.2. Let $T$ be an invertible isometry of $L_{1}(S, \Sigma, \mu)$. Then $T^{* *} \eta$ is a pure charge if $\eta$ is.

Proof. For any $\nu \in M(S, \Sigma, \mu)$ we have $\left(T^{* *}\right)^{-1} \nu \in M(S, \Sigma, \mu)$ (with $\left.\frac{d}{d \mu}\left(T^{* *}\right)^{-1} \nu=T^{-1} \frac{d \nu}{d \mu}\right)$. Hence

$$
\left\|T^{* *} \eta+\nu\right\|=\left\|\eta+\left(T^{* *}\right)^{-1} \nu\right\|=\|\eta\|+\left\|\left(T^{* *}\right)^{-1} \nu\right\|=\left\|T^{* *} \eta\right\|+\|\nu\|
$$

and we apply Lemma 4.1(i).

Proposition 4.3. Let $T$ be an invertible isometry of $L_{1}(S, \Sigma, \mu)$. If $f \in L_{1}$ satisfies $\sup _{n}\left\|\sum_{k=1}^{n} \frac{T^{k} f}{k}\right\|_{1}<\infty$, then $f \in G(T) L_{1}$. If $T$ is also mean ergodic, then $\sum_{k=1}^{\infty} \frac{T^{k} f}{k}$ converges in $L_{1}$-norm.

Proof. We identify $f$ with the measure it defines. We apply Proposition 3.5 to $T^{* *}$ and obtain an element $\eta \in L_{1}^{* *}$ with $G\left(T^{* *}\right) \eta=f$. We decompose $\eta=\eta_{1}+\eta_{0}$ with $\eta_{1}$ countably additive and $\eta_{0}$ a pure charge. Since $T$ is an invertible isometry, $\left(T^{* *}\right)^{k} \eta_{0}$ is a pure charge for every $k$, so Lemma 4.1(ii) shows that $G\left(T^{* *}\right) \eta_{0}$ is a pure charge. Since $G\left(T^{* *}\right) \eta_{1}$ is countably additive, $f=G\left(T^{* *}\right) \eta_{1}+G\left(T^{* *}\right) \eta_{0}$ implies $f=G(T) g$ with $g=d \eta_{1} / d \mu$.

If $T$ is also mean ergodic, $G(T) L_{1}=G(T) \overline{(I-T) L_{1}}$ and Proposition 3.3 yields the convergence.

COROLlarY 4.4. Let $\theta$ be an invertible measure preserving transformation of a probability space $(S, \Sigma, \mu)$. If $f \in L_{1}(S, \mu)$ satisfies $\sup _{n}\left\|\sum_{k=1}^{n} \frac{f \circ \theta^{k}}{k}\right\|_{1}<\infty$, then the one-sided EHT $\sum_{k=1}^{n} \frac{f \circ \theta^{k}}{k}$ converges a.e. and in $L_{1}$-norm. 
Proof. The operator $T f=f \circ \theta$ is a mean ergodic invertible isometry, so the result follows by combining Proposition 4.3 with [6, Theorem 1.2].

The following theorem deals with general probability preserving transformations which may not be invertible. To deal with this contingency, we first assume that the measure space in question is a Lebesgue space; in this setting the existence of an invertible probability preserving transformation (also on a Lebesgue space) which has the original one as a factor (the natural extension) is well-known (e.g. [4, p. 240]).

TheOREM 4.5. Let $\theta$ be a measure preserving transformation of a Lebesgue probability space $(S, \Sigma, \mu)$ with $\theta(S)=S$. If $f \in L_{1}(S, \mu)$ satisfies

$$
\sup _{n}\left\|\sum_{k=1}^{n} \frac{f \circ \theta^{k}}{k}\right\|_{1}<\infty,
$$

then the one-sided EHT $\sum_{k=1}^{n} \frac{f \circ \theta^{k}}{k}$ converges a.e. and in $L_{1}$-norm.

Proof. For $f \in L_{1}(\mu)$ put $T f:=f \circ \theta$. Then $T$ is an isometry of $L_{1}(\mu)$, mean ergodic because $\mu$ is finite.

When $\theta$ not invertible, there exists an invertible probability preserving $\hat{\theta}$ on $(\hat{S}, \hat{\Sigma}, \hat{\mu})$ with a factor map $\pi: \hat{S} \rightarrow S$ such that $\hat{\mu}\left(\pi^{-1} A\right)=\mu(A)$ and $\pi \circ \hat{\theta}=\theta \circ \pi$, which yields $\pi \circ \hat{\theta}^{n}=\theta^{n} \circ \pi$ for $n \geq 0$. We put $\hat{T} \hat{g}=\hat{g} \circ \hat{\theta}$ for $\hat{g} \in L_{1}(\hat{S}, \hat{\mu})$. For $f \in L_{1}(S, \mu)$ define $\tilde{f}=f \circ \pi$; the identity $\widetilde{1_{A}}=1_{\pi^{-1} A}$ yields $\|\tilde{f}\|_{1}=\|f\|_{1}$, and we obtain

$$
\widetilde{T^{k} f}=\widetilde{f \circ \theta^{k}}=\left(f \circ \theta^{k}\right) \circ \pi=(f \circ \pi) \circ \hat{\theta}^{k}=\tilde{f} \circ \hat{\theta}^{k}=\hat{T}^{k} \tilde{f},
$$

so $\left\|\sum_{k=1}^{n} a_{k} T^{k} f\right\|_{1}=\left\|\sum_{k=1}^{n} \widetilde{a_{k}} T^{k} f\right\|_{1}=\left\|\sum_{k=1}^{n} a_{k} \hat{T}^{k} \tilde{f}\right\|_{1}$.

It follows that if $f \in L_{1}(S, \mu)$ satisfies $[9]$, then $\sup _{n}\left\|\sum_{k=1}^{n} \frac{\hat{T}^{k} \tilde{f}}{k}\right\|_{1}<\infty$, and by Proposition 4.3 . $\sum_{k=1}^{n} \frac{\hat{T}^{k} \tilde{f}}{k}$ converges in $L_{1}(\hat{\mu})$-norm. Now

$$
\left\|\sum_{k=j}^{n} \frac{T^{k} f}{k}\right\|_{1}=\left\|\sum_{k=j}^{n} \frac{\hat{T}^{k} \tilde{f}}{k}\right\|_{1}
$$

shows that $\left\{\sum_{k=1}^{n} \frac{T^{k} f}{k}\right\}$ is Cauchy in $L_{1}(\mu)$, so converges in norm. The a.e. convergence follows from the norm convergence by [6, Theorem 1.2].

Acknowledgments. Parts of this research were carried out during the second author's visit to Ben-Gurion University, supported by its Center for Advanced Studies in Mathematics, and the third author's visit to Equipe ERIM of the University of New Caledonia. These authors are grateful for the hospitality of their hosts. 


\section{References}

[1] I. Assani and M. Lin, On the one-sided ergodic Hilbert transform, in: Contemp. Math. 430, Amer. Math. Soc., 2007, 21-39.

[2] J. T. Campbell, Spectral analysis of the ergodic Hilbert transform, Indiana Univ. Math. J. 35 (1986), 379-390.

[3] G. Cohen and M. Lin, The one-sided ergodic Hilbert transform of normal contractions, in: Characteristic Functions, Scattering Functions and Transfer Functionsthe Moshe Livsic Memorial Volume, Birkhäuser, Basel, 2009, 77-98.

[4] I. Cornfeld, S. Fomin and Ya. Sinai, Ergodic Theory, Springer, Berlin, 1982.

[5] M. Cotlar, A unified theory of Hilbert transforms and ergodic theorems, Rev. Mat. Cuyana 1 (1955), 105-167.

[6] C. Cuny, On the a.s. convergence of the one-sided ergodic Hilbert transform, Ergodic Theory Dynam. Systems 29 (2009), 1781-1788.

[7] Y. Derriennic and M. Lin, Fractional Poisson equations and ergodic theorems for fractional coboundaries, Israel J. Math. 123 (2001), 93-130.

[8] N. Dunford and J. T. Schwartz, Linear Operators. Part I: General Theory, WileyInterscience, New York, 1958.

[9] V. F. Gaposhkin, Spectral criteria for existence of generalized ergodic transforms, Theory Probab. Appl. 41 (1996), 247-264.

[10] P. Halmos, A nonhomogeneous ergodic theorem, Trans. Amer. Math. Soc. 66 (1949), $284-288$.

[11] E. Hille and R. Phillips, Functional Analysis and Semigroups, Amer. Math. Soc., Providence, RI, 1957.

[12] S. Izumi, A non-homogeneous ergodic theorem, Proc. Imp. Acad. Tokyo 15 (1939), 189-192.

[13] U. Krengel, Ergodic Theorems, de Gruyter, Berlin, 1985.

[14] M. Lin and R. Sine, Ergodic theory and the functional equation $(I-T) x=y$, J. Operator Theory 10 (1983), 153-166.

[15] K. Yosida, Functional Analysis, 6th ed., Springer, Berlin, 1980.

[16] K. Yosida and E. Hewitt, Finitely additive measures, Trans. Amer. Math. Soc. 72 (1952), 46-66.

[17] A. Zygmund, Trigonometric Series, 2nd ed., Cambridge Univ. Press, Cambridge, 1969 .

Guy Cohen

Department of Electrical Engineering

Ben-Gurion University

Beer Sheva 84105, Israel

E-mail: guycohen@ee.bgu.ac.il

Christophe Cuny

Equipe ERIM

University of New Caledonia

Nouméa, New Caledonia

E-mail: cuny@univ-nc.nc

Michael Lin

Department of Mathematics

Ben-Gurion University

Beer Sheva 84105, Israel

E-mail: lin@math.bgu.ac.il 